\title{
Designing Memory Probes to Inform Dialogue
}

Wenn-Chieh Tsai

Faculty of Engineering and IT

University of Technology Sydney

Sydney, Australia

wenn-chieh.tsai@uts.edu.au

\section{Daniel Orth}

Faculty of Engineering and IT

University of Technology Sydney

Sydney, Australia

daniel.orth@uts.edu.au
Elise van den Hoven

Faculty of Engineering and IT

University of Technology Sydney

elise.vandenhoven@uts.edu.au

Department of Industrial Design

Eindhoven University of

Technology

e.v.d.hoven@tue.nl

Duncan of Jordanstone College of

Art and Design

University of Dundee

Australian Research Council's

Centre of Excellence in Cognition

and its Disorders

Macquarie University

\section{Abstract}

To investigate the phenomenon that occurs during

interactions between used objects and autobiographical memories, which are both ever-changing and imbedded with personal significance, an adapted probing method capable of managing these complex qualities is

needed. This pictorial is our attempt to find a nuanced indication of how probes could go beyond common usage to facilitate complex felt experience, and how probes can be used in less prescriptive ways to instead promote reminiscent dialogues that are rich and open to interpretation for both participants and researchers. It illustrates our exploration into potential Memory Probes and how this might be done that reflects the value we see in creating restrictions or limitations in technologymediated interactions to encourage active participation by users in social acts such as memory creation and remembrance.

\section{Authors Keywords}

Autobiographical memory, cherished object, design

probe, participatory inquiry paradigm, reminiscence, traces of use.

\section{ACM Classification Keywords}

H.5.m. Information interfaces and presentation (e.g., HCI): Miscellaneous. 


\section{Introduction}

Our daily interactions with our (physical or digital) belongings can not only leave traces of use on them but also memories in our minds. The goal of this project is to understand the relationship between traces on cherished objects and human memories, which are both constantly changing and entangled with personal significance. To achieve that, we adopted a participatory inquiry paradigm that considers what is intrinsically valuable in human life [6]. This paradigm focuses on the relationship between participants and researchers and what can be known in this relationship. For conducting research in this way, efforts must be made to foster dialogue between the two through mutual understanding and concessions during their knowledge co-construction.

We devised Memory Probes to sensitize research participants as they revisit their belongings and aid them in collecting materials for reminiscent dialogues with us. They were not only tools used to investigate participants' experiences but also an intermediate that was iteratively updated to convey messages between our participants and us as researchers. This mediated dialogue provides a reciprocal understanding process for each other [14]. For us, the used probes and accompanied interviews provided insights of participants' values and interpretations of

research questions that helped us reframe our approach in the design process. For participants, the probes provided an opportunity to re-interact with their cherished objects from a new perspective.

This pictorial showcases parts of our process and findings from our inquiry into the traces of use on objects and human memories. It focuses on the design and use of our research artifacts, the Memory Probes, to illustrate how we translated our design concept into the features of probes and how these probes were interpreted and "co-performed" [8] with research participants. The constructive design research [9] in this pictorial was comprised of one exploratory study and one field study, each with different aims. The exploratory study aimed to discover possibilities for using the probes and to make adjustments that followed feedback from experienced interaction design researchers. The field study investigated personal memories related to cherished belongings with traces of use. In the field study, the Memory Probes were adapted and deployed into participants' living spaces for one to two weeks and subsequently brought to interviews to prompt discussion.

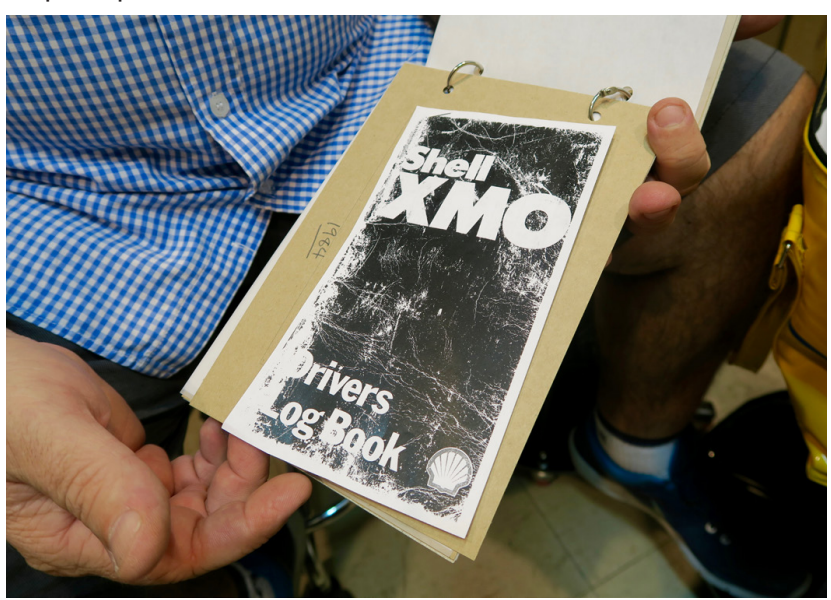

A participant reminisces about his life at university through the maintenance logs of his first car. 


\section{Probing and Memory}

The use of probes as a research method is often intended to sensitize participants, heightening their awareness of their everyday routines and encouraging them to reflect on their preferences $[12,13]$. Cultural probes rely heavily on the designers' interpretations whilst design probes emphasize a co-constructed understanding through a continuous dialogue $[4,11,17]$, an approach we adopt in our inquiry. In this dialogue, the users sharing their experiences and life stories are not simply reporting but rather actively co-constructing meaning with their objects and the researchers [10].

While people are using probes, they are making metaphoric interpretations of their lives and belongings from their current perspectives. They choose what and how they want to show and embody these interpretations into collected information. This process resonates with the constructive nature of autobiographical memory and provides an opportunity for memory reconstruction. Although the information collected by probes are low-fidelity and fragmented, this "uncertainty" provides designers with valuable insights into participants' lives and the consequential inspiration they provide $[4,5]$.

Few studies have provided detail on how users interact with probe kits $[1,5,17]$. Little is known about how the perceived form and function of design probes moderate users presentation and how the expressive quality of collected information impacts a designer's understanding. To address our research goals, we aimed to sensitize and shift people's attention from the object as a whole to its explicit or even implicit traces of use. We also wanted to keep individuals values and personal significance in the inquiry process. Instead of relating specifically to a question or context as thematic probes [17], we devised our probes based on the pre-existing senses, e.g. sight, hearing, and touch.
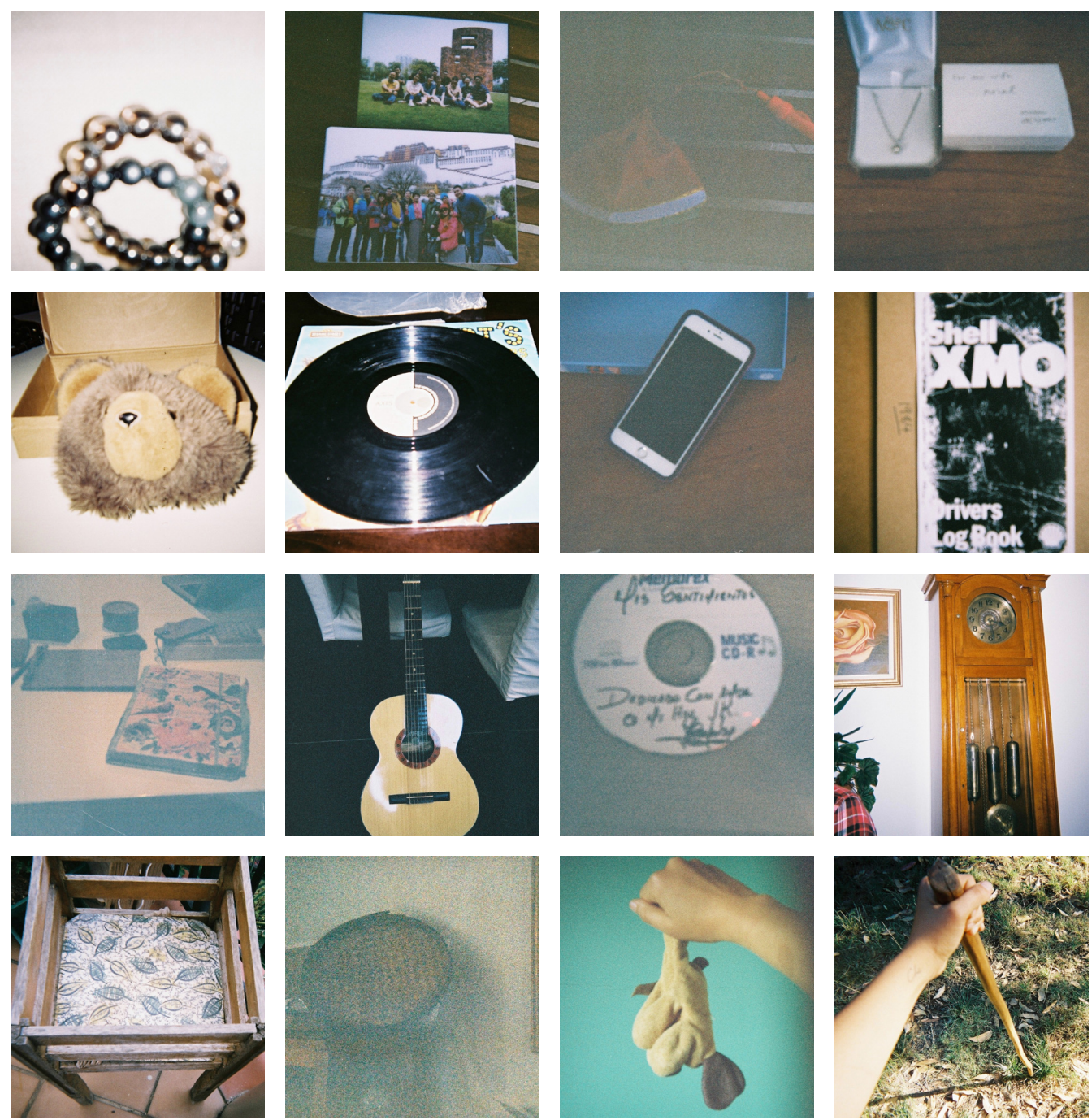

Photos of participants' cherished belongings that were developed and later returned as a gift. 


\section{Design Rationale}

We aimed to create activities and materials that not only elicited inspirational responses from participants, but also engaged with them through

meaningful interactions

in which their personal preferences and personhood could manifest. Intrinsically, we attempted to explore how they determined what was important and why.

We created pairs of values to guide the design process of both the probe materials and the activities conducted with participants. Through adopting the participatory inquiry paradigm, we as co-subjects were reflexively involved in the constructive design research just as our participants were. The Memory Probes were also materials for our own empathetic engagement in dialogue with the participants. In other words, the Memory Probes were our cherished objects. The traces left by participants on the probes were entrances into their life stories.

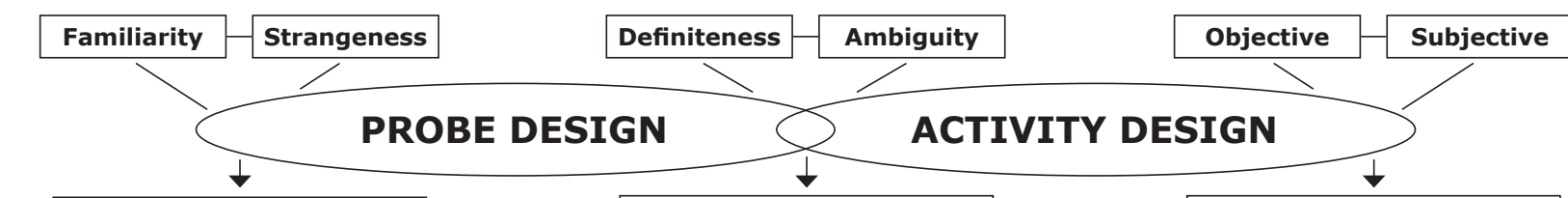

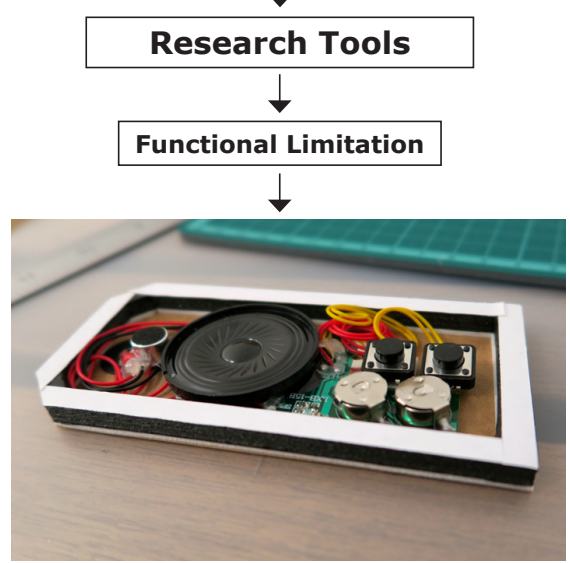

A gift-tag recorder with only 10 sec capacity.

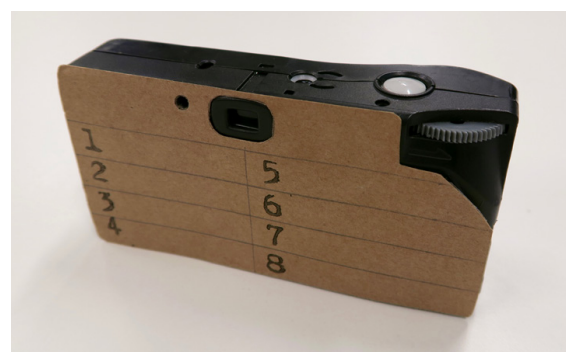

An analog camera with only 8 shots.

Familiarity and strangeness of tool use. The former provides good affordance and locus of control and the latter increases awareness and sensibility

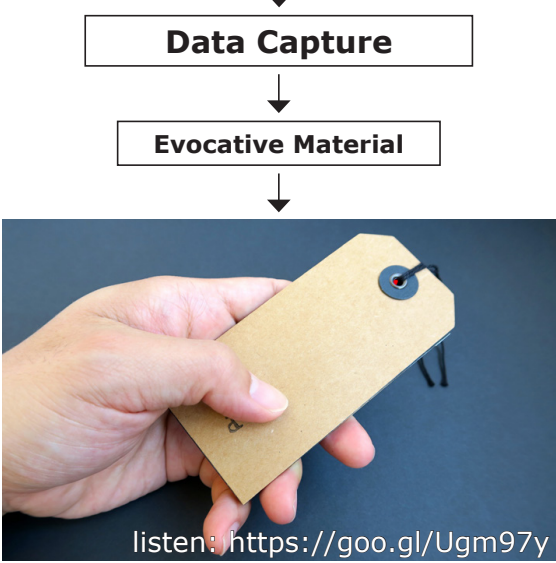

Lo-fi quality of recorded sound.

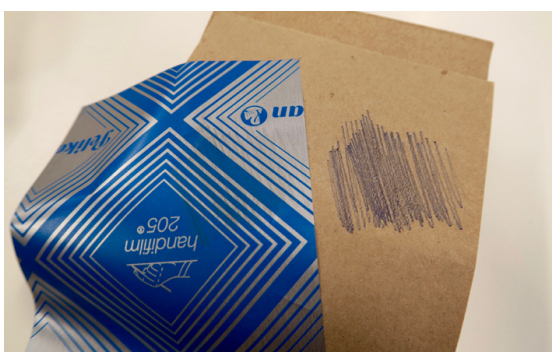

Vague copy of texture.

Definiteness and ambiguity of data capture. The former provides more information and the latter provides alternative and enriched interpretation $[3,15]$.
Data Interpretation $\downarrow$ Slow Understanding

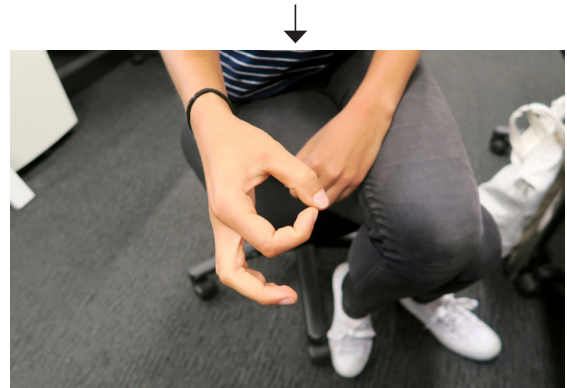

Embodying the feature with memory.

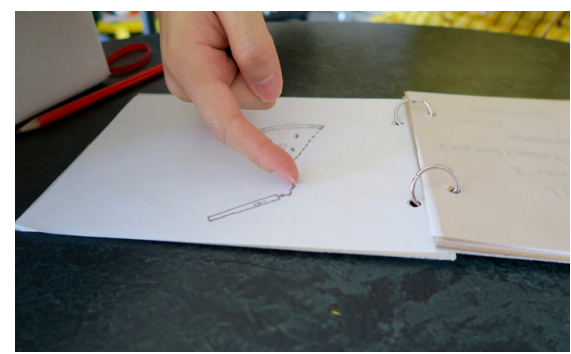

Co-performing with the probes in-situ.

Objective and subjective reality of interpretation. Researchers and participants construct knowledge together in democratic dialogues as co-researchers and as co-subjects [6]. 


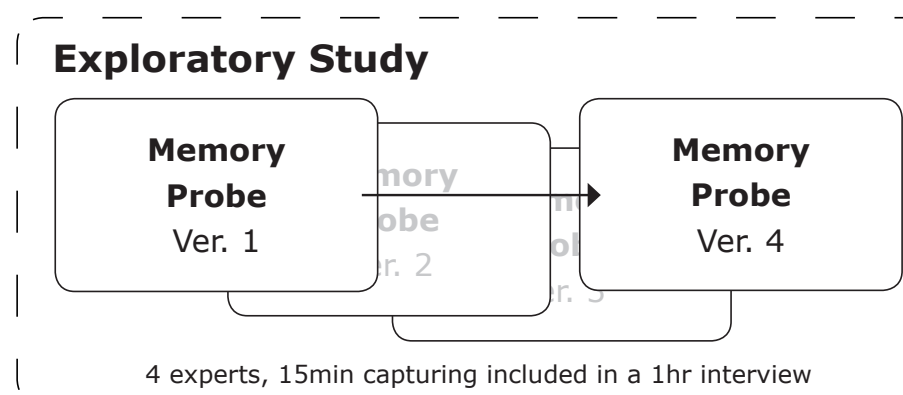

\section{Exploratory Study: Dialogues with Experts}

Four interaction design researchers who had relevant

experience participated in our exploratory study. We

focused on discussion that explored the ways of using our

Memory Probes and acquiring preliminary understanding

about how limitation on functionality and evocative

quality of the captured information provoked participants

remembering experiences. Participating researchers

were encouraged to use the package creatively without

constraint and at the conclusion of the interview were

invited to reflect on the activities and provide comments

or suggestions. We improved the probes after each

interview by removing and adding tools and materials

following the feedback provided and our own insights.

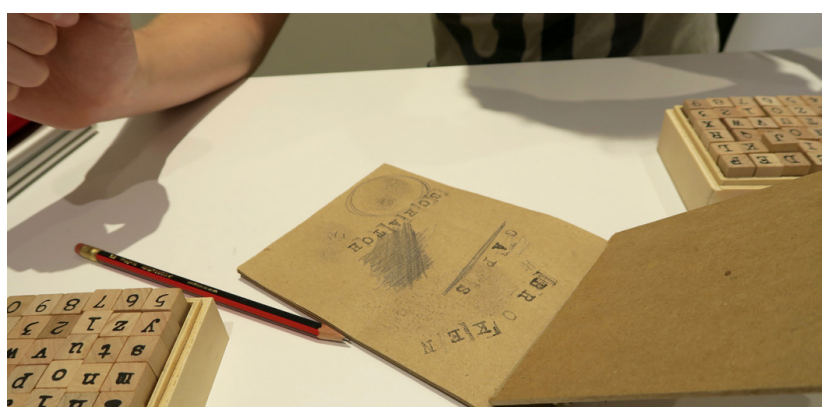

A researcher providing feedback on the Memory Probe kit.

I Field Study

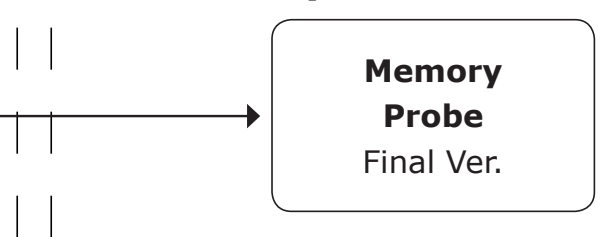

| $\mid 7$ participants, $1-2$ weeks capturing and $1 \mathrm{hr}$ final interview |

\section{Field Study: Dialogues with Participants}

With the final version of the Memory Probes, we

conducted a field study with seven participants in which we asked them to interact with our revised probes in their homes. This was then followed by a one-hour elicitation interview. The participants were between 22 to 78 years old and consisted of three women and four men who were from diverse backgrounds and life experiences.

We focused on understanding the relation between human traces on cherished objects and remembering experiences as well as how our Memory Probes mediate interactions between these two. This pictorial presents the findings related to the usage of the Memory Probes.

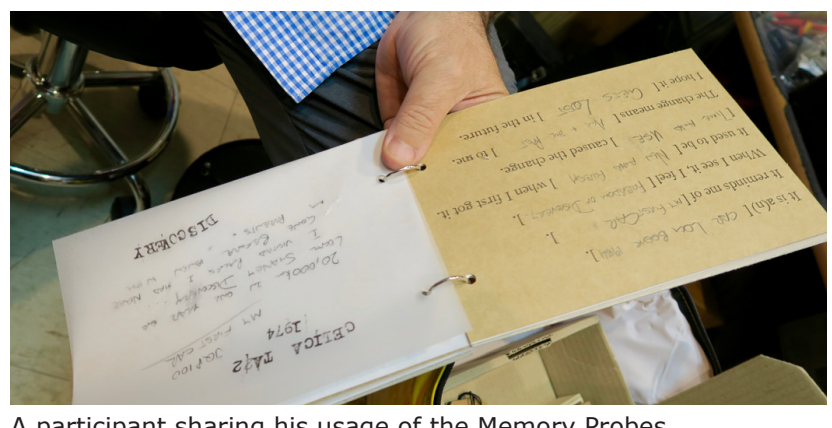

A participant sharing his usage of the Memory Probes. 


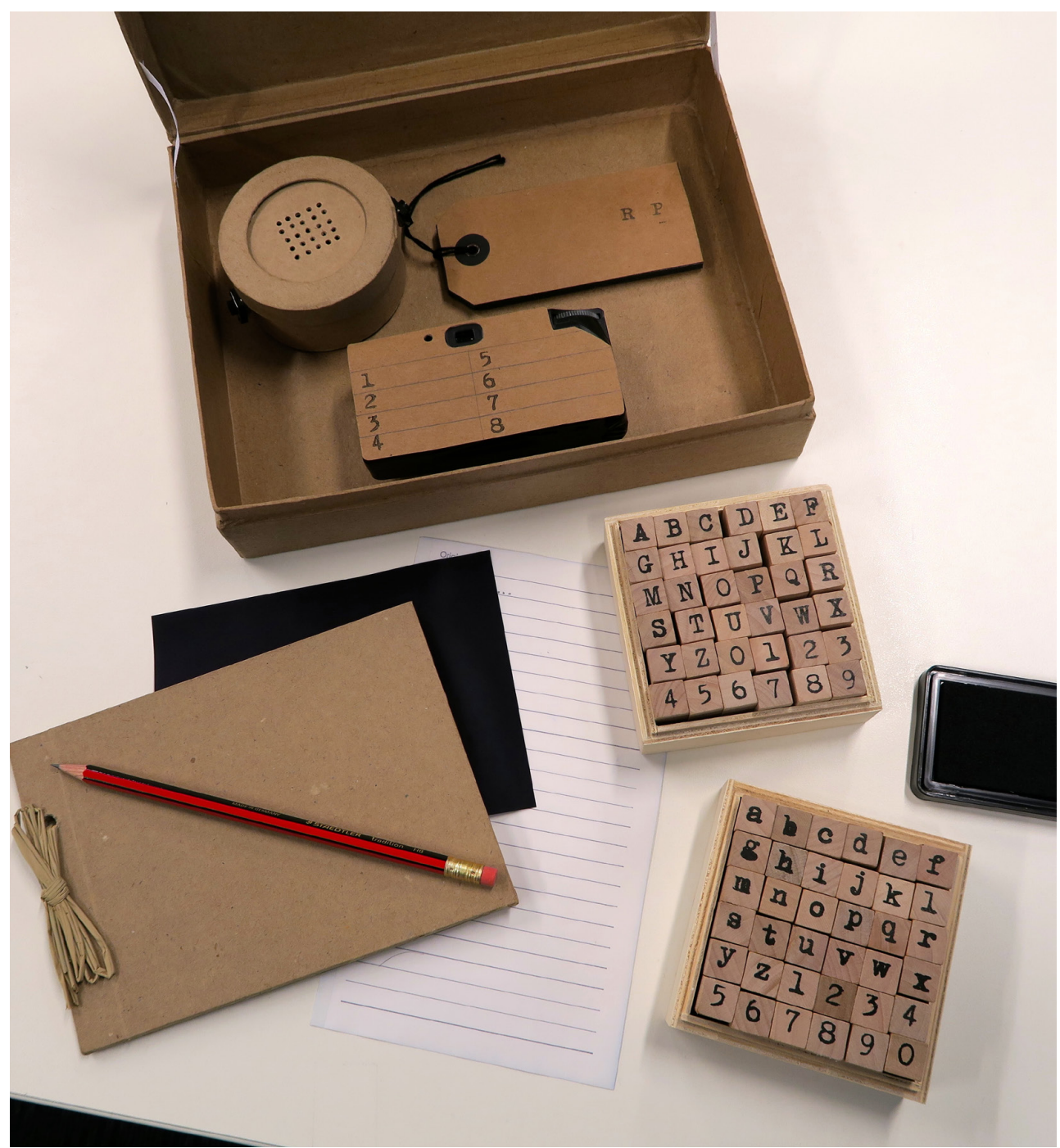

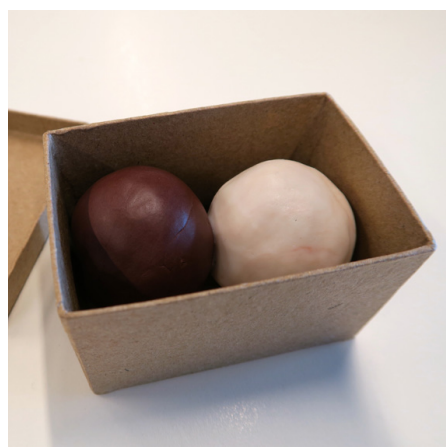

Exploratory Probe Kit In the exploratory study, we provided two probes with similar functions but with slightly different forms, materials, or expressions for each type of modality. We aimed to see if this intertool contrast could implicitly sensitize the experts to

the slight differences of affordance and quality of captured information as well as elicit more discussion on preferences and choices in the exploratory interview.

For example, the first version

of our probes included a

disposable camera and a kraft notepad for users to capture

what they visually perceive.

The sheets of the kraft notepad can be used to record

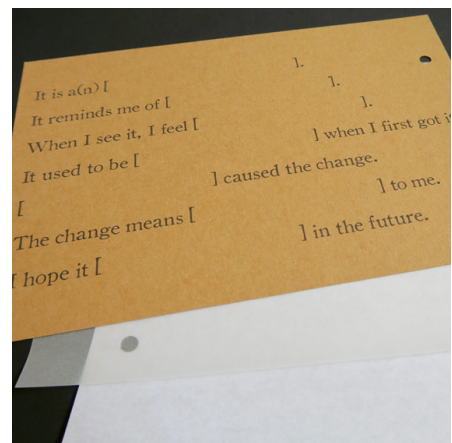

surface patterns of objects by rubbing or sketching. SoundTag and SoundBox were designed for capturing auditory properties of objects and their environments by recording a 10-second clip of sound. Two sets of lowercase and uppercase letters and numbers stamps were provided

to conceptualize and type words in the kraft notepad. One carbon paper and one carbonless paper, which are used to duplicate written strokes, were also included into the package with a pencil. The kit was continually updated during the exploratory study with some materials such as clay and kraft card with prompts, tracing paper, and thin writing pad paper added. 


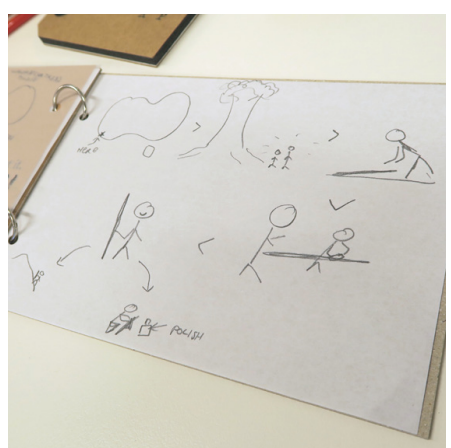

Probe Activities

There were two probe activities in this study, capturing and introducing. In capturing, participants had to choose their cherished objects that had traces of use. They were provided with our Memory Probes to revisit and capture properties and memories relating to the chosen objects. Instead of giving prescriptive instructions, we provided tools and materials for sensitizing users' free association in a more perceptual way. The probes were introduced and demonstrated according to their capabilities of capturing what people see, hear, touch, and think. In the activity of introducing, participants brought the

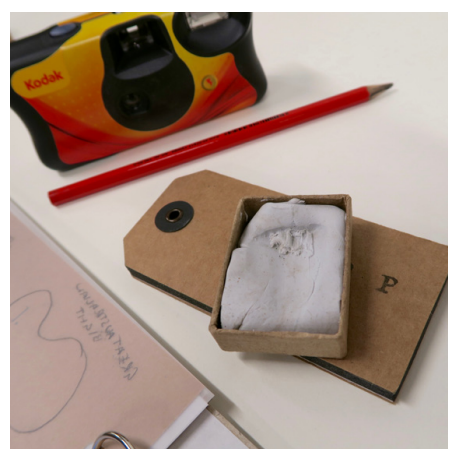

captured information, but not the objects, to individua interviews. They shared their memories relating to the objects and the traces, using the captured information except the photos taken with the disposable cameras. The photos were later developed as a token of appreciation

for their involvement. During interviews, we chose not to see the real objects and photos but construct our understanding through the fragmented lo-fi information and the participants' narratives.

We intended for this slow understanding to help us focus on the participants' points of views and to co-construct an intersubjective reality of interpretation.

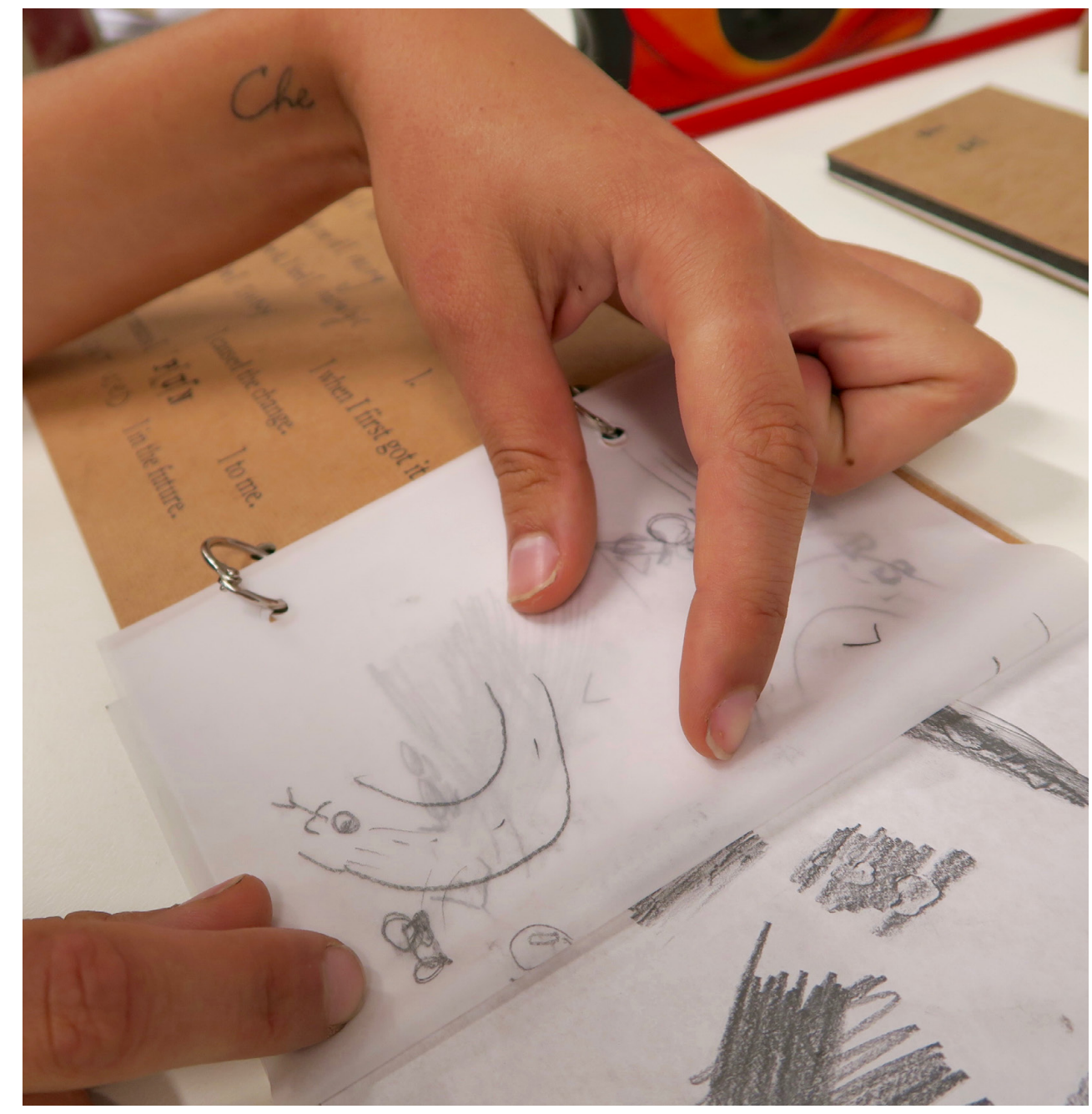

The participant uses pencil rubbings of her helmet alongside a hand-drawn storyboard to narrate her experience of falling off her motorcycle. 

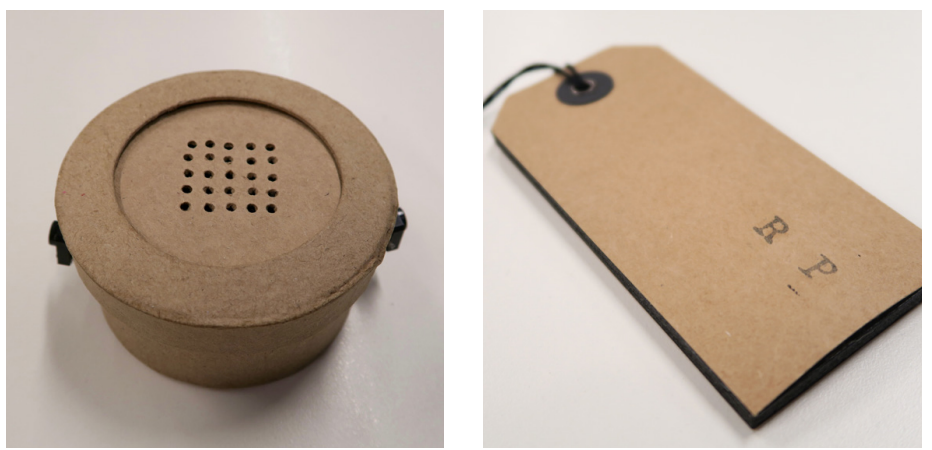

\section{Probe Refinements}

Participants' willingness to use the probes was not only dictated by its functionality or form, but also its perceived quality of expression. For example, the lo-fi and muffled sound from SoundTag was deemed "forgivable", however the louder and echoed sound from SoundBox was seen as "intolerable."
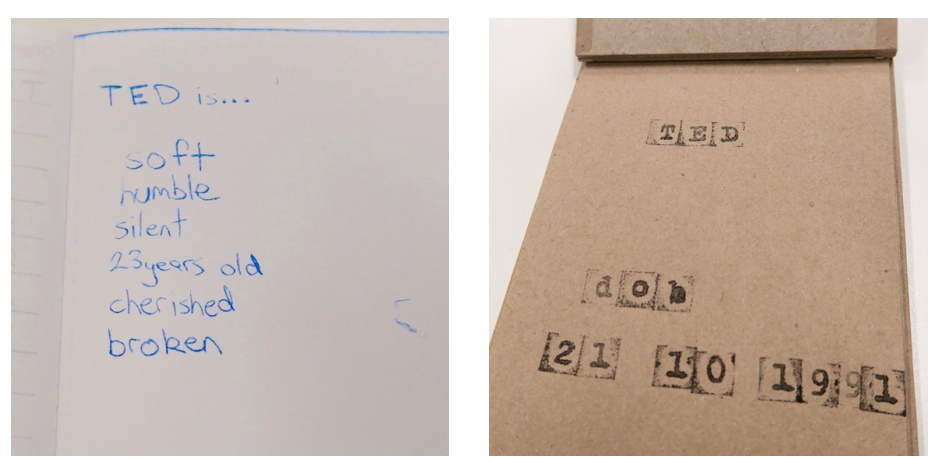

The stamps were used to give emphasis to a phrase but were too slow to type a full sentence. We chose the uppercase alphabet and numbers stamps to promote keyword typing in contrast to writing.

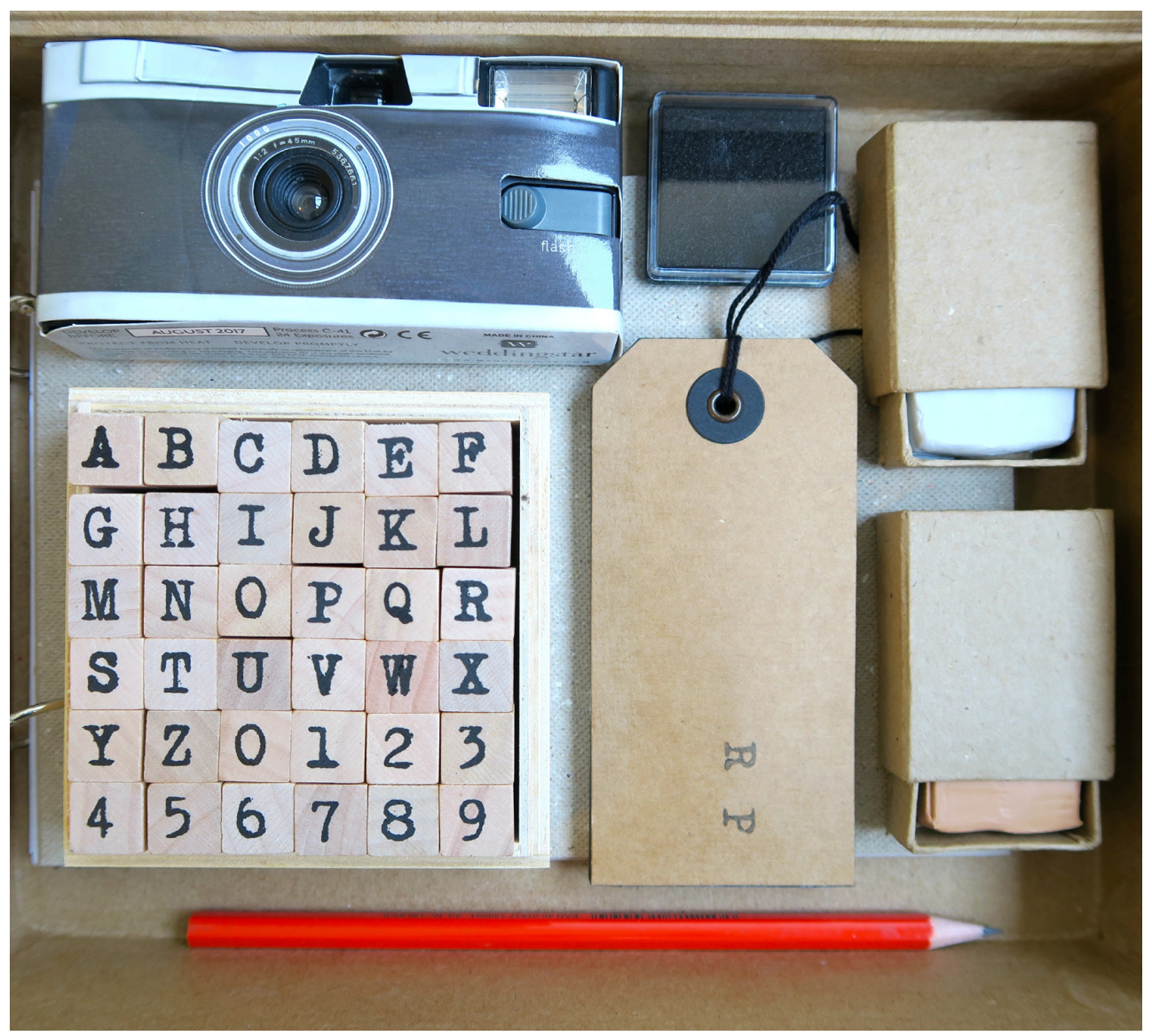

Final Probe Kit

The final probes that were used in the field study contained a disposable camera, an ink pad, a set of uppercase alphabet stamps, a SoundTag, two pieces of clay, a pencil, and a loose-leaf photo album containing three types of sheets; kraft card, tracing paper, and thin writing pad paper. 


\section{Examples of Memory Probe Usage}

The participants demonstrated several ways of using the probes to support their narratives. Some were related to the limited

functionality and evocative materials we chose for the designed probes. Other methods of use involved the participants' reenacting aspects of their narratives with their prepared materials

or things that were within arms reach in the interview. In many cases it was an embodied performance with objects and an engaged sense making process through multi-modal apparatuses. In the following section, we provide examples of the various ways in which participants chose to use our Memory Probes.

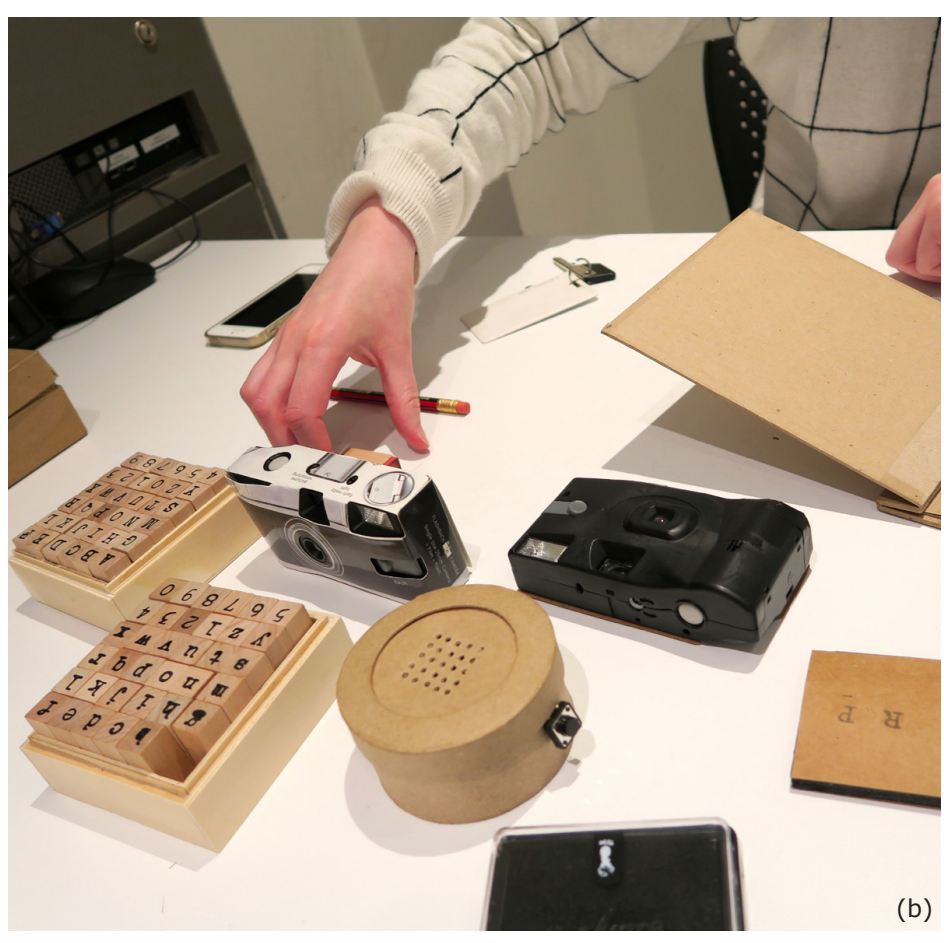

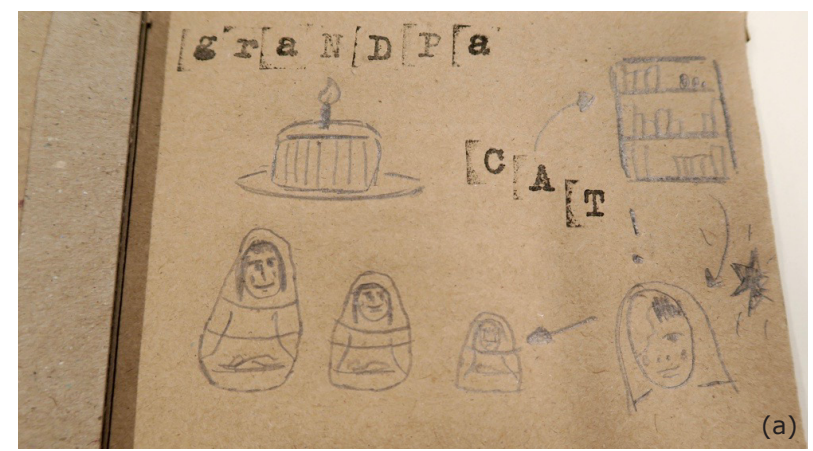

Re-constructing

Story Scenes

Some information was not

captured from the object itself

but purposely structured to

support their storytelling. For

example, (a) drawing a

storyboard or (b) dropping a

stamp on the desk to simulate

the sound of a wooden doll

crashing into the ground.
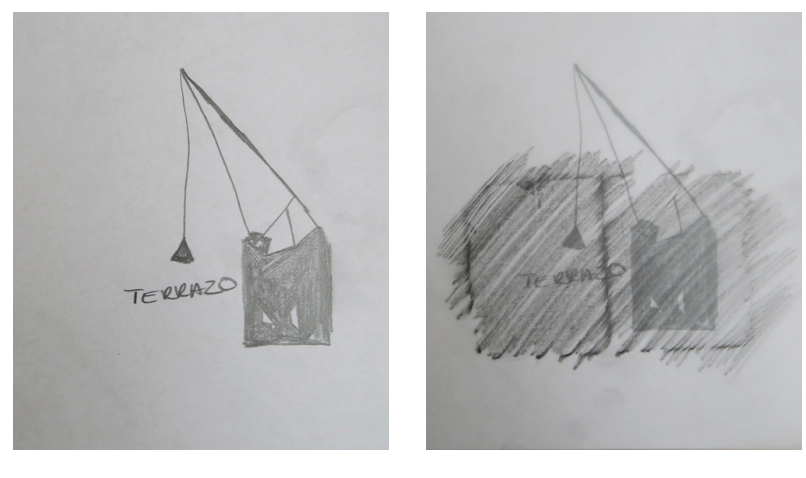

Layers of Meaning

The sketching or rubbing traces on the writing paper could be

seen through the tracing paper.

It layers different meanings

of an object. For example, a

participant accidently folded a

given and cherished business

card from his father, who was

working in a construction

company.
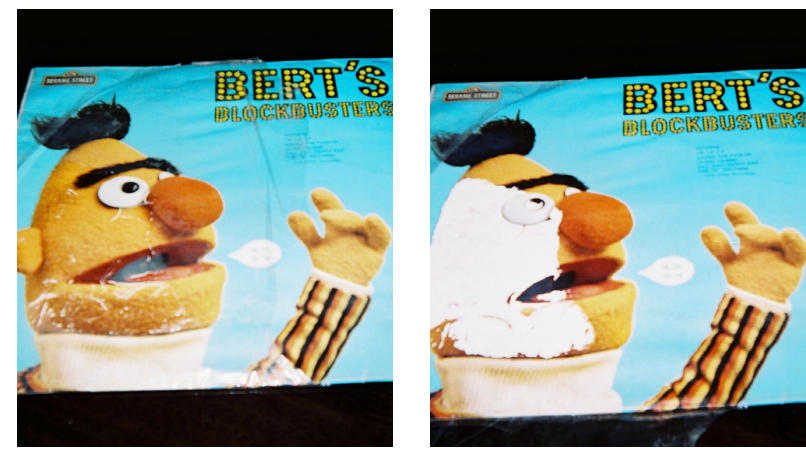

Transition Effects

Two photos or sketched images can be used to show the

effects of use or deterioration.

For example, these two

pictures show that a

participant's LP album cover

had been peeled away with

the color adhered to the

protecting sheet due to a long

history of use. 
Materializing Traces and Materializing Concepts Some uses focused on directly capturing and representing traces on objects as well as those recollected memories about objects and traces. The given fidelity of the captured information was an agent of abstraction nudging towards users' interpretations.

It relied on both the material expressivity and functionality of probes. For example, a stamp could be used to create sound effects by its wooden material; on the other hand, it could be used to stamp a letter and assemble a word to express a concept by its given function. The former leverage the benefit of ambiguity of its evocative materiality, and the latter relied on the definite function provided by the

tool. Its materiality provided additional affordance beyond its functional use. The usage of the probes showed how different pairs of design values were effectively intertwined in the probes and creatively used in reminiscent dialogues.
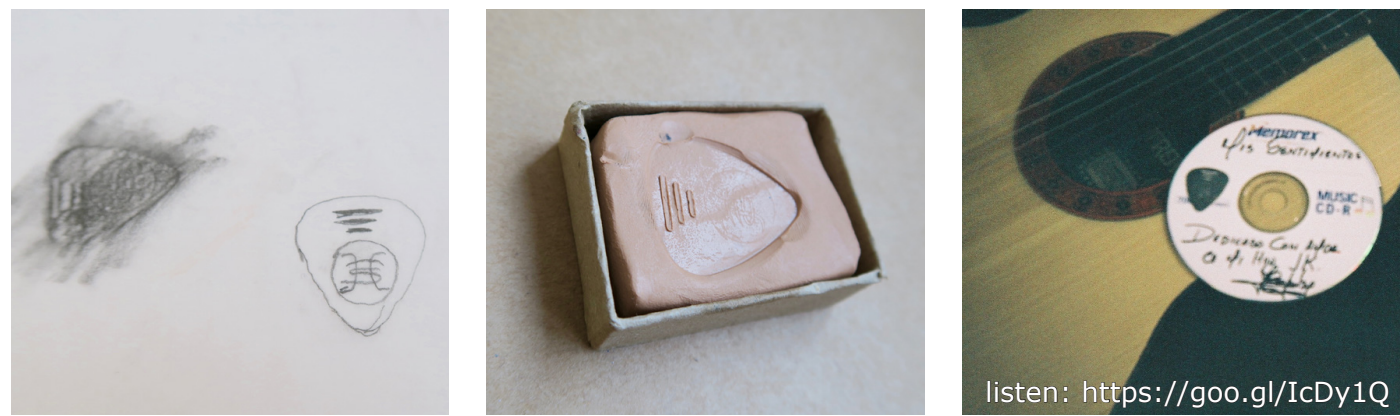

A participant used our probes to describe the guitar pic that reflects his and his ex-wife's shared love of music.
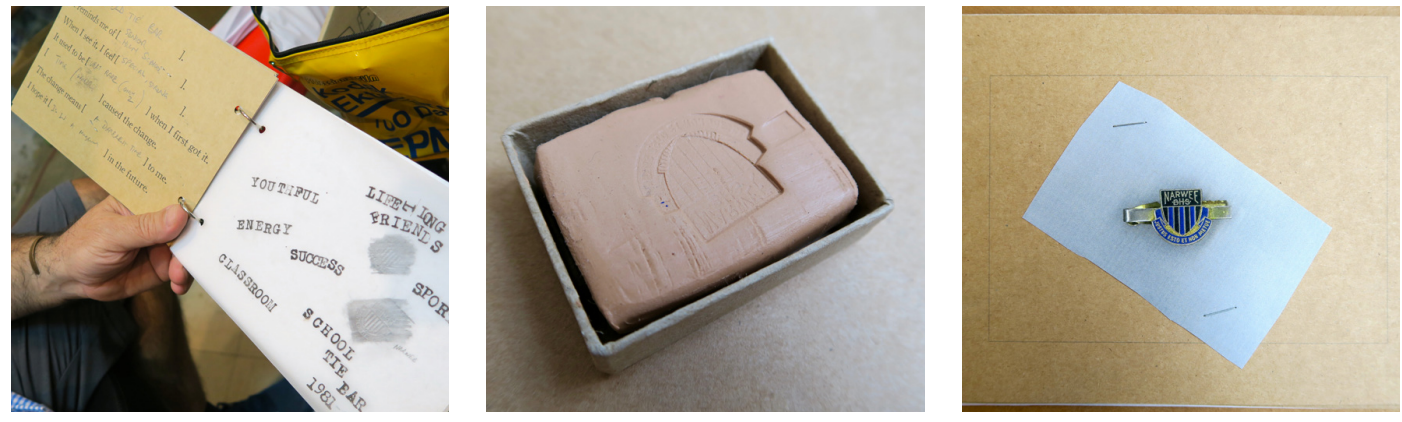

Another participant used our probes to re-construct the memories cued by his high school tie bar.
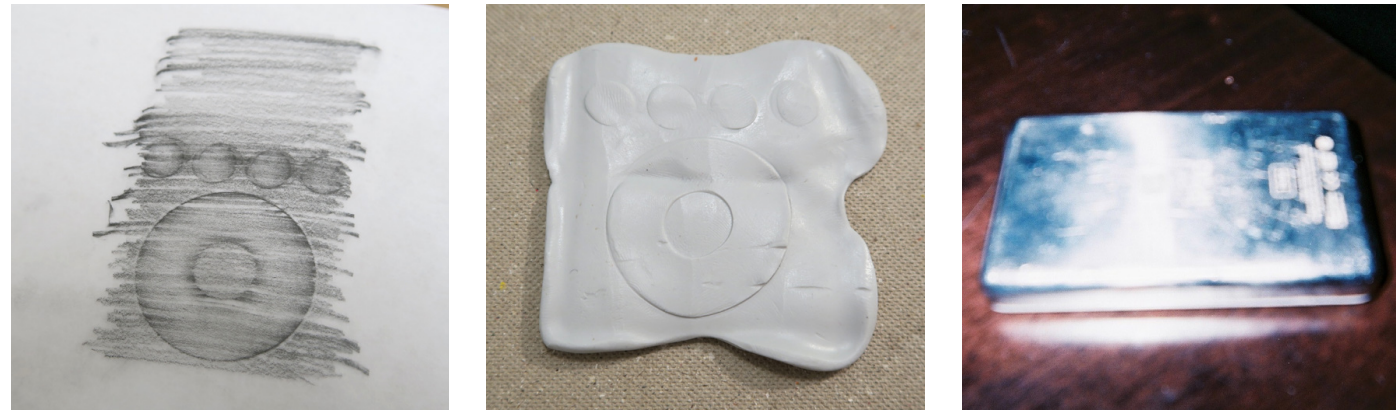

A third participant used our probes to capture multiple details of his beloved yet now broken gift from his wife. 
Embodied Performance with Objects

For making sense of their stories, participants relied on the materials they prepared for the focuses of stories and enacted these stories with the objects they could reach during the interview session.

\section{Multimodal Performance}

In this case, participant $\mathrm{G}$

combined different methods of

communication to compensate

for the limited information

she captured to share a story

about her cherished bamboo

tray. All these together led the

interviewer down a memory

lane of her terrible time in

the hospital with her late

husband, their friends' wishes, and her feelings.
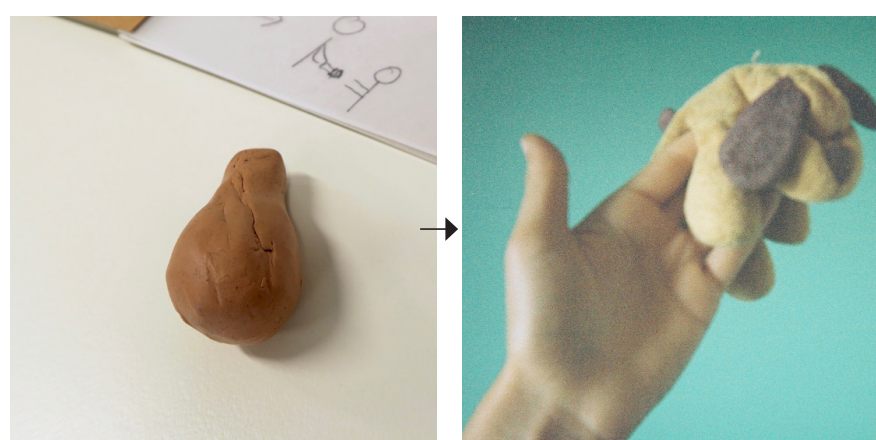

Our probes were used to show the form of the plush-toy dog's leg.

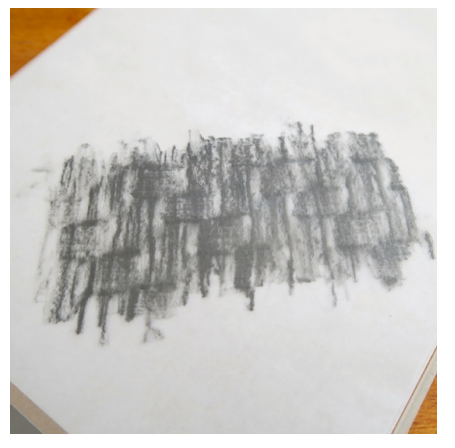

Materialized Traces With pencil rubbings of the

bamboo pattern, $\mathrm{G}$ described the strengthened timber base that was added by her husband.

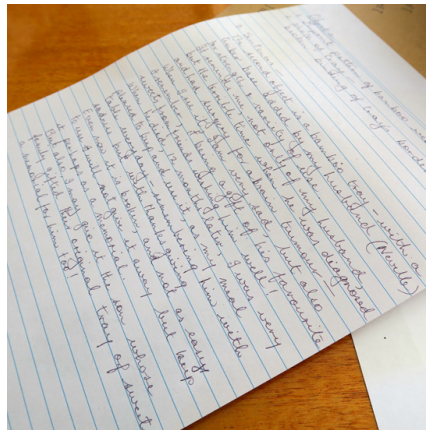

\section{Reconstructed Stories}

In her written note, $\mathrm{G}$ expressed the feelings she experienced

when she used it - "rememberin him with sadness but with thanksgiving".
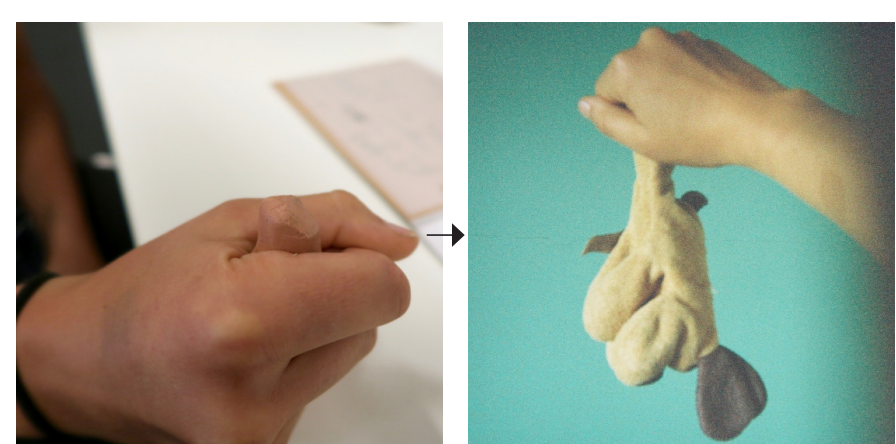

These probes were then used to show how she often carried the dog.

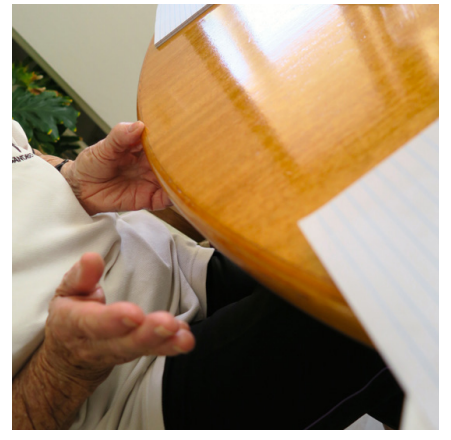

Embodied Performance Without showing the object or developed photos, $\mathrm{G}$ was

demonstrated the broken binding of the tray's border with the table and her gesture.

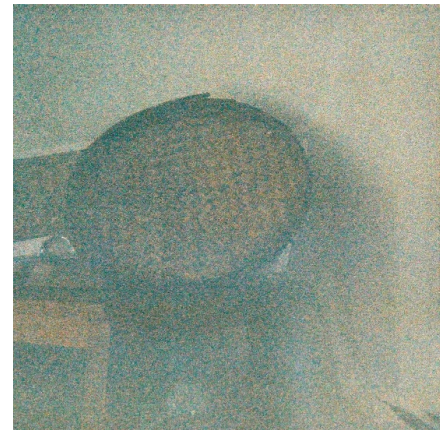

Cherished Bamboo Tray G's bamboo tray was a gift from friends wishing her late husband well when he was in hospital. It was used as her everyday meal tray afterward. 


\section{Discussion}

Although we encouraged the participants to use all the probes in the package, some chose to only use a selection of tools and materials. This could be attributed to several reasons, such as their cherished object and the probe provided having no corresponding properties that could be used in their co-performances. Other reasons raised by participants included the fragility of their cherished objects and the unfamiliar nature of certain tools. The exploratory nature of the task instructions also created a sense of agency for participants to decide for themselves how they wish to engage with the materials and tools, as well as the level of detail of objects' features.

We saw our participants as co-researchers tasked with a role of self-interpretation rather than merely following a prescribed order of activities. Adopting this role was found to be difficult by some of our participants without any prior learning phase included in the study. We argue that research aiming to create co-constructed dialogues between researcher and participant must therefore acknowledge the varying characteristics of participants and undergo an iterative process in the design of probe materials and activities to alleviate the heightened expectations of participants. Our use of paired values to guide our design process assisted us in creating research tools and activities that were flexible in their adoption, allowing participants to engage with tools at a point on the paired-values spectrum that they found familiar.

Reflections on our Slow Understanding

Without seeing their cherished objects in the interview, participants had to narrate rather than describe their experiences. We prompted them with their collected information and waited for their initiation of storytelling and information revealing without our own judgments.
This was based on the common ground that they were in control of our knowing because they decided what information to show and hide for making sense of their stories. They had to help us understand what they chose to let us know and value. We were not only designing interactions for our participants but also for ourselves because we as co-subjects were participating and engaging in this curiosity-driven understanding process [16]. In this mutual understanding, limiting technology played a positive role in the dialogue that fostered our shared external and internal remembering [7].

\section{Conclusion}

This pictorial illustrates how our probes find a dynamic balance between familiarity and strangeness of probe use through functional limitations; definiteness and ambiguity of data capture through evocative materials; objective and subjective reality of interpretation through a slow understanding process. We present a nuanced indication of how probes could go beyond common usage to facilitate complex felt experience, and how probes can be used in less prescriptive ways to instead promote reminiscent dialogues that are rich and open to interpretation for both participants and researchers. The design of our probe materials reflect the value we see in creating restrictions or limitations in technology-mediated interactions to

encourage active participation by users in social acts such as memory creation and remembrance.

\section{Acknowledgements}

This research was supported by the Postdoctoral Research Abroad Program of Taiwan Ministry of Science and Technology, and STW VIDI grant number 016.128.303 of The Netherlands Organization for Scientific Research (NWO) awarded to Elise van den Hoven (UTS HREC Ref. \#2015000427). 


\section{References}

1. Kirsten Boehner, Janet Vertesi, Phoebe Sengers, and Paul Dourish. 2007. How HCI interprets the probes. In Proceedings of the SIGCHI Conference on Human Factors in Computing Systems (CHI '07), ACM Press, 1077-1086.

2. Anthony Dunne and Fiona Raby. 2001. Design Noir: The Secret Life of Electronic Objects. Birkhäuser.

3. William W. Gaver, Jacob Beaver, and Steve Benford. 2003. Ambiguity as a resource for design. In Proceedings of the SIGCHI Conference on Human Factors in Computing Systems (CHI '03), ACM Press, 233-240

4. William W. Gaver, Andrew Boucher, Sarah Pennington, and Brendan Walker. 2004. Cultural probes and the value of uncertainty. interactions 11, 5: 53-56.

5. Connor Graham, Mark Rouncefield, Martin Gibbs, Frank Vetere, and Keith Cheverst. 2007. How probes work. In Proceedings of the 19th Australasian conference on Computer-Human Interaction: Entertaining User Interfaces (OZCHI '07), ACM Press, 29-37.

6. John Heron and Peter Reason. 1997. A participatory inquiry paradigm. Qualitative Inquiry 3, 3: 274-294.

7. Elise van den Hoven and Berry Eggen. 2014. The cue is key. Zeitschrift für Psychologie 222, 2: 110-117.

8. Elvin Karana, Elisa Giaccardi, Niels Stamhuis, and Jasper Goossensen. 2016. The tuning of materials: a designer's journey. In Proceedings of the 2016 ACM Conference on Designing Interactive Systems (DIS '16), ACM Press, 619-631.

9. Ilpo Koskinen, John Zimmerman, Thomas Binder, Johan Redstrom, and Stephan Wensveen. 2011. Design Research Through Practice: From the Lab, Field, and Showroom. Elsevier.
10. Tuck Wah Leong, Peter Wright, Frank Vetere, and Steve Howard. 2010. Understanding experience using dialogical methods. In Proceedings of the 22nd Conference of the Computer-Human Interaction Special Interest Group of Australia on ComputerHuman Interaction (OZCHI '10), ACM Press, 256-263.

11. Tuuli Mattelmäki. 2006. Design Probes. University of Art and Design, Helsinki.

12. Ine Mols, Elise van den Hoven, and Berry Eggen. 2014. Making memories: a cultural probe study into the remembering of everyday life. In Proceedings of the 8th Nordic Conference on Human-Computer Interaction: Fun, Fast, Foundational (NordiCHI '14), ACM Press, 256-265.

13. Daniela Petrelli, Elise van den Hoven, and Steve Whittaker. 2009. Making history: intentional capture of future memories. In Proceedings of the SIGCHI Conference on Human Factors in Computing Systems (CHI '09), ACM Press, 1723-1732.

14. Elizabeth B.-N. Sanders and Pieter Jan Stappers. 2014. Probes, toolkits and prototypes: three approaches to making in codesigning. CoDesign 10, 1 : 5-14.

15. Phoebe Sengers and Bill Gaver. 2006. Staying open to interpretation: engaging multiple meanings in design and evaluation. In Proceedings of the 6th conference on Designing Interactive systems (DIS '06), ACM Press, 99-108.

16. Wenn-Chieh Tsai, Amy Yo Sue Chen, Sheng-Yang Hsu, and Rung-Huei Liang. 2015. CrescendoMessage: interacting with slow messaging. In Proceedings of the 2015 International Association of Societies of Design Research Conference (IASDR '15), 2078-2095.

17. Jayne Wallace, John McCarthy, Peter C. Wright, and Patrick Olivier. 2013. Making design probes work. In Proceedings of the SIGCHI Conference on Human Factors in Computing Systems (CHI '13), ACM Press, 3441-3450. 